\title{
Asthma with bronchial hypersecretion: expression of mucins and toll-like receptors in sputum and blood
}

This article was published in the following Dove Press journal:

Journal of Asthma and Allergy

12 October 2017

Number of times this article has been viewed

\begin{abstract}
Astrid Crespo-Lessmann', Eder Mateus ${ }^{1,2}$, Montserrat Torrejón', Alicia Belda', Jordi Giner', Silvia Vidal', Oriol Sibila', Vicente Plaza'

'Service of Pneumology, Hospital de la Santa Ceu i Sant Pau, Institute of Sant Pau Biomedical Research (IBB Sant Pau), Universitat Autònoma de Barcelona, Centro de Investigación Biomédica en Red de Enfermedades Respiratorias (CIBERES), ${ }^{2}$ Department of Immunology, Hospital de la Santa Creu i Sant Pau, Institut d'Investigació Biomédica Sant Pau, Barcelona, Spain
\end{abstract}

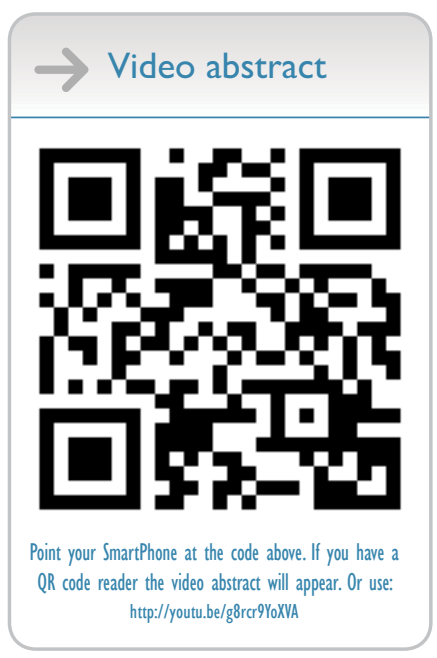

Correspondence: Astrid Crespo-Lessmann Department of Respiratory Medicine, Hospital de la Santa Creu i Sant Pau, Carrer Mas Casanovas 90, Barcelona 0804I, Spain

Tel +34935565972

Fax +3493556560

Email acrespo@santpau.cat
Abstract: Asthma with bronchial hypersecretion is a type of asthma that is poorly studied. Its pathogenesis is not well understood, but is probably related to innate impaired immunity, particularly with toll-like receptors (TLRs) and secretory mucins (MUC).

Objectives: 1) Define the clinical and inflammatory phenotype of asthma with bronchial hypersecretion of mucus. 2) Compare the type of mucin present in induced sputum (IS) of patients with and without bronchial hypersecretion. 3) Determine the expression of TLRs in IS and blood of asthmatics with and without bronchial hypersecretion.

Materials and methods: Cross-sectional study which included 43 non-smoking asthmatic patients without bronchiectasis, 19 with bronchiectasis, and 24 without bronchial hypersecretion. All patients underwent the following: IS, spirometry, fractional exhaled nitric oxide, prick test, total immunoglobulin E (IgE), and blood albumin. Analysis of mucins was determined by ELISA and expression of TLR2 and TLR4 by flow cytometry. The level of asthma control was determined by the Asthma Control Test (ACT) questionnaire and quality of life was assessed by the reduced version of the Asthma Quality of Life Questionnaire (mini-AQLQ).

Results: Asthmatics with bronchial hypersecretion were significantly older (62.6 years vs 48.5 years; $p=0.02$ ); had greater severity (persistent severe asthma $94.7 \%$ vs $29.2 \%$; $p=0.000$ ); a higher proportion of nasal polyposis $(36.8 \%$ vs $8.3 \%$; $p=0.022)$; less control of asthma $(73.7 \%$ vs $8.3 \%$; $p=0,000)$; a higher proportion of asthma with negative prick test $(68.4 \%$ vs $16.6 \%$; $p=0.001$ ), and lower levels of $\operatorname{IgE}(113.4 \mathrm{IU} / \mathrm{mL}$ vs $448 \mathrm{IU} / \mathrm{mL} ; p=0.007)$, compared with asthmatics without bronchial hypersecretion. Significant differences were observed neither in the expression of TLRs 2 and 4 in inflammatory cells of IS or peripheral blood, nor in the expression of mucins between both groups.

Conclusion: Asthma patients with bronchial hypersecretion have more severe and uncontrolled disease, with poor quality of life as well as a non-allergic inflammatory phenotype. Within the mechanisms involving these differences, it does not appear that mucins and TLRs play an important role.

Keywords: asthma, mucins, inflammation, induced sputum, toll-like receptor

\section{Introduction}

Bronchial inflammation in asthmatic patients with chronic airflow obstruction and bronchial hypersecretion, superimposed on phenotype 5 of diseases of the airway described by Weatherall et al, ${ }^{1}$ differs from the usual classic allergic eosinophilic asthma. However, available information about this type of asthma is scarce. It is well known that the mucus of asthmatic patients is characterized by its higher viscosity and may play an important role in the pathogenesis of the obstruction that accompanies 
the disease and in fatal asthma attacks. Some studies have reported that this could be related to the different mucin profile (ie, MUC2, MUC5B>MUC5A, MUC5AC) that these patients present. ${ }^{2-4}$ However, it is unknown whether the profile of mucins secreted by asthmatic patients with bronchial hypersecretion differs from those of asthmatics not suffering from bronchial mucus hypersecretion. Mucins (MUC) are glycoproteins that are part of airway mucus; they are responsible of its viscoelastic properties and play an important antimicrobial and anti-inflammatory role, acting as a first line of immunological defence. ${ }^{5}$ MUC are composed of alpha and beta subunits and form a complex heterodimer. The alpha subunit acts in cell adhesion, and the beta unit in cellular signaling. Increasingly, the beta subunit is related to the activation of so-called toll-like receptors (TLRs). TLRs are a family of trans-membrane proteins responsible for the recognition of pathogen-associated molecular patterns, molecules characteristic of microorganisms such as lipopolysaccharides, flagellins, mannans, or nucleic acids of viruses and bacteria. This recognition leads to rapid production of cytokines, providing an adaptive and lasting response against the pathogen. ${ }^{6}$ The relationship between bronchial hypersecretion, mucins, and activation of TLRs would open a new field in the pathogenesis of this form of the disease, as it would establish a relationship of innate immunity and respiratory infections with bronchial hypersecretion.

It is known that bronchial mucus hypersecretion leads to impaired mucociliary clearance and abnormal bacterial implantation, and promotes mucus plugs in the airways. ${ }^{7}$ Despite these characteristics observed in the clinical followup of some asthma patients, few studies have included this variant of asthma, and none have studied the distinguishing characteristics of these patients. Morinaga et al and Kraft et al associated infections with bronchial hypersecretion, demonstrating increased mucin production (MUC5AC) in cultured epithelial cells of asthmatic patients when stimulated by Chlamydia and M. pneumoniae. ${ }^{8,9}$ They also showed that mucin production decreased when they exposed bronchial epithelial cells to macrolide ${ }^{9}$ and that the effect (in the case of $M$. pneumoniae infection) disappears when TLR-2 and an NF-KB inhibitor are added. ${ }^{10}$ This fact implicates TLR-2 in the initial inflammatory response leading to exacerbations of asthma produced by $M$. pneumoniae and relates TLRs to mucins. ${ }^{10,11}$ In the same vein, another study recently showed that MUC1 specifically plays a role during inflammatory bacterial infection of the airways, not only because of TLR-5 signal suppression, ${ }^{12}$ but also because it involves other TLRs, such as $2,3,4,7$, and $9 .{ }^{12}$ It is suspected that
MUC1, in addition to suppressing the activation of NF-KB (at the TRAF6-TAK1 complex level), can stimulate TLRs signaling. ${ }^{13}$

This study had the following objectives: 1) Define the clinical and inflammatory phenotype of asthma with bronchial hypersecretion of mucus. 2) Compare the type of mucin present in induced sputum (IS) of patients with and without bronchial hypersecretion. 3) Determine the expression of TLRs in the IS and in the peripheral blood of asthmatics with and without bronchial hypersecretion.

\section{Materials and methods Study design}

This cross-sectional study carried out in a tertiary referral university hospital in Barcelona, Spain. The aim of the study was to define the clinical and inflammatory phenotype of asthma with bronchial hypersecretion of mucus, compare the types of mucin present in IS in patients with and without bronchial hypersecretion, and determine the expression of TLRs in the IS and peripheral blood between the two groups.

\section{Subjects}

Male and female subjects aged $>18$ years, non-smokers, without bronchiectasis, who fulfilled the diagnostic criteria of asthma were consecutively enrolled from the Asthma Unit outpatient clinic of our institution. We defined asthma as a history of variable respiratory symptoms with evidence of variable expiratory airflow limitation. All patients had a positive bronchodilator test or a positive methacholine challenge test documented in the case history. Asthma severity was defined according to the Global Initiative for Asthma Management (GINA). ${ }^{14}$ All patients included in the study underwent a chest computed tomography (CT), in which the presence of bronchiectasis or other non-asthmatic pathology was ruled out (lung cancer, pulmonary tuberculosis, sarcoidosis, nephropathy, and rheumatic or liver disease). Moreover, patients were excluded from the study if they had a respiratory tract infection and/or required the use of oral corticosteroids within 30 days prior to inclusion; had a significant comorbidity that could affect the study results in the physician's judgment; or had a cognitive impairment that could limit their comprehension of the subject under study or their ability to participate.

\section{Ethics approval and consent to participate}

This study was conducted in accordance with the principles of the Declaration of Helsinki (18th World Medical 
Assembly) and was approved by the Independent Ethics Committee of Hospital de la Santa Creu i Sant Pau (approval number COD:IIBS-ROF-2011-106). All patients gave written informed consent to participate in the study. Subjects were included upon receipt of written informed consent and personal identification data were anonymized. ClinicalTrials. gov Identifier: NCT02948153. Registered October 18, 2016. Retrospectively registered.

\section{Assessments and study procedures}

In 43 asthmatic patients, demographic and clinical variables were recorded. On the same day, the following determinations or tests were performed: FeNO, forced spirometry, inflammatory cell count in IS, serum total IgE levels, eosinophil count in the peripheral blood, and skin prick test to common aeroallergens in our environment. Patients also completed a Spanish validated version of the Asthma Control Test (ACT) ${ }^{15}$ and the reduced version of the Asthma Quality of Life Questionnaire (mini-AQLQ). ${ }^{16}$ Asthmatics patients with bronchial hypersecretion were defined as those who expectorated daily for at least 3 months for a minimum period of two consecutive years, without attribution to any other cause or disease. All patients underwent microscopic analysis of IS, and the presence of associated bacteria in the sputum was ruled out (exclusion criterion).

FeNO was measured before spirometry using electrochemical equipment (NO Vario Analyzer, Berlin, Germany) and an expiratory maneuver providing a sustained $50 \mathrm{~mL} / \mathrm{s}$ flow from total lung capacity, according to the 2005 recommendations of the American Thoracic Society/European Respiratory Society (ERS). ${ }^{17}$ A significant increase in FeNO was considered if the FeNO value was equal or higher than 50 parts per billion (ppb). ${ }^{18}$ Spirometry was undertaken using a Daptospir-600 spirometer (Sibelmed, Barcelona, Spain) by an experienced pulmonary function technician and according to the 2003 recommendations of the Spanish Society of Pneumology and Thoracic Surgery (SEPAR), ${ }^{19}$ with $\mathrm{FEV}_{1}$ in the reference range when $\geq 80 \%$ predicted. ${ }^{20}$ IS samples were harvested according to ERS consensus protocol $^{21}$ and processed for flow cytometry and conventional readouts. ${ }^{22}$ Briefly, mucus plugs were manually selected and homogenized in $0.1 \%$ dithiothreitol (DTT; Calbiochem, San Diego, CA, USA) for $15 \mathrm{~min}$, then washed and filtered through a 41- $\mu \mathrm{m}$ pore nylon mesh (Millipore, Membrane solutions, Dallas, TX, USA). Cell viability and quality were determined by trypan blue exclusion dye. Samples with less than $10^{6}$ cells/g, less than $40 \%$ viability, and greater than $20 \%$ squamous epithelial cells were excluded. Slides with $5 \times 10^{4}$ cells were stained with Diff-Quik kit (Polysciences Europe
GmbH, Eppelheim, Germany), Cell count and differential leukocyte count were done following references that were previously established. ${ }^{23}$ We suspended $10^{6}$ sputum cells in 1 $\mathrm{mL}$ staining buffer $[3 \%$ bovine serum albumin in phosphatebuffered saline (PBS)] and delivered into a $100-\mu \mathrm{L}$ tube for flow cytometry analysis. Patients with a neutrophil count $\geq 61 \%$ were classified as having neutrophilic asthma, those with eosinophil sputum count $\geq 3 \%$ as eosinophilic asthma, those with a neutrophil count $<61 \%$ and a eosinophil sputum count $<3 \%$ as paucigranulocytic asthma, and those with a neutrophil count $\geq 61 \%$ and a eosinophil sputum count $\geq 3 \%$ as mixed asthma. ${ }^{24}$

Serum total IgE was measured by ImmunoCAP using the UniCAP 250 system (Phadia AB, Uppsala, Sweden), with increased levels for values $>160 \mathrm{IU} / \mathrm{mL}$. Skin prick testing was conducted according to a standard procedure, with positive results when the wheal diameter was $>3 \mathrm{~mm} .^{25}$ The 100- $\mu \mathrm{L}$ sample of peripheral blood was immunostaining for flow cytometry and erythrolysed with TQ Prep Sample Preparation and COULTER PrepPlus 2 (Beckman Coulter, Miami, FL, USA).

To objectively assess the level of asthma control, we used the ACT. This is a simple, five-item questionnaire that measures the degree of control of the asthmatic patient assigned to their illness over the last 4 weeks. The answers for each question are assigned $1-5$ points, resulting in a total score ranging from 5 (worst possible control) to 25 (the best possible control). A score more than 20 corresponds to "controlled asthma," and less than 19 points to "not wellcontrolled asthma." 15 High doses of inhaled steroids were considered as beclomethasone dipropionate (or equivalent) $\geq 1,000 \mu \mathrm{g} /$ day. ${ }^{26}$ To determine the quality of life related to asthma, we used the mini-AQLQ. ${ }^{16}$

\section{Analysis of mucin by ELISA}

Mucin levels were determined by Enzyme Linked Immunosorbent Assay (ELISA), following manufacturer's instructions. MUCIN 1 and MUCIN 5B were purchased from Wuxi Donglin Sci\&Tech Development Co., Ltd., MUCIN 2 was purchased from Elabscience Biotechnology Co., Ltd., and MUCIN 5AC was purchased from Life Science Inc. MUCIN detection ranges were MUC $1(0.15-10 \mathrm{ng} / \mathrm{mL})$, MUC2 (0.313-20 ng/mL), MUC5AC (78-5,000 pg/mL), and MUC5B $(0.625-40 \mathrm{ng} / \mathrm{mL})$.

\section{TLRs analysis by flow cytometry}

Blood and sputum cells were blocked with mouse serum and immunostained with anti-TLR4 PE (clone HTA125), 
anti-CD66b PE (G10f5), and anti-CD14 PE/Cy7 (clone M5E2) from Biolegend (San Diego, CA, USA); anti-TLR2 Alexa Fluor 488 (clone 11G7) and anti-CD125 PE (A14) from BD Biosciences (Eembodegen, Belgium); and antiCD16 PE (3G8), anti-CD45 PE (MEM28), anti-CD16 FITC (3G8), anti-CD66b FITC (B13.9), and anti CD45 PE/Dy-647 (MEM28) from Immunotools (Oldenburg, Germany). ${ }^{22}$ Samples were incubated in the dark for $15 \mathrm{~min}$ at room temperature. Then, cells were washed with $2 \mathrm{~mL}$ staining buffer and centrifuged for $5 \mathrm{~min}$ at $1,750 \mathrm{rpm}$. Supernatants were decanted, the cells resuspended in $300 \mu \mathrm{L}$ staining buffer, and the samples were stored at $4^{\circ} \mathrm{C}$ in the dark until analysis on the flow cytometer within $2 \mathrm{~h}$ from preparation.

Flow cytometric data acquisition was performed with the FC500 equipment (Beckman Coulter, Pasadena, CA, USA). Ten thousand events were counted for all sample runs. Gating for discrimination of leukocytes was based on CD45 expression versus side-light scatter. The mean fluorescence intensity (MFI) of the cells stained with control antibody was subtracted from the MFI of the cells stained with receptor antibodies to provide a measure of receptorspecific fluorescence.

\section{Statistical analysis}

Categorical variables were expressed as frequencies and percentages, and continuous variables as mean and standard deviation (SD). Comparisons between both groups were undertaken with the Student's $t$ test for quantitative variables and the chi-square $\left(\chi^{2}\right)$ test or the McNemar's test for categorical variables. Statistical significance was set at $p<0.05$. Statistical analysis was conducted with SPSS version 18.0 for Windows (Chicago, IL, USA).

\section{Results}

\section{Clinical phenotypes of asthmatics} patients with and without bronchial hypersecretion

Compared to the non-hypersecretory group, hypersecretory asthmatics patients were older, had greater disease severity, increased bronchial obstruction, greater use of high doses of inhaled steroids, a higher proportion of nasal polyposis, less control of asthma, lower score on the MiniAQLQ, a greater number of emergency room visits in the previous year, a greater need for short courses of oral corticosteroids in the previous year, a higher proportion of asthma with negative prick test, lower levels of IgE, lower albumin levels in peripheral blood, and lower levels of lymphocytes in IS (Table 1).

\section{Inhaled treatment}

In the asthma group with bronchial hypersecretion, $21 \%$ used budesonide and formoterol; 52.6\% used fluticasone and salmeterol; and 26.4\% used beclomethasone and formoterol. In the asthma group without bronchial hypersecretion, $12.5 \%$ used budesonide and formoterol; $20.8 \%$ used fluticasone and salmeterol; $33.3 \%$ used beclometasone and formoterol; and $33.4 \%$ only received salbutamol $(p=0.018)$. On the other hand, $47.36 \%$ of asthma patients with bronchial hypersecretion had anticholinergics associated as maintenance treatment. None of the non-hypersecretors had this treatment. The difference between the two groups is due to the greater severity of the disease that asthma patients with bronchial hypersecretion experience.

\section{Mucin expression}

Mean mucin expression of all asthmatics patients $(\mathrm{N}=43)$ was MUC1: $114.72 \mathrm{ng} / \mathrm{mL}$ (standard deviation [SD] 59.30); MUC2: 24.42 ng/mL (SD 19.75); MUC5AC: 456.18 pg/mL (SD 1123.3); and MUC5B: $1.24 \mathrm{ng} / \mathrm{mL}$ (SD 4.04). Thus, in all asthmatics patients in the study $(\mathrm{N}=43)$, MUC 1 was very high, followed by MUC 2; however, MUC5AC and MUC 5B were within the reference values (see the section on mucin detection ranges in the analysis of mucin by ELISA).

The expression of mucins MUC1, MUC2, MUC5AC, and MUC5B in patients with and without bronchial hypersecretion is shown in Table 2. No significant differences in levels of mucin present in IS were observed between the two groups of asthmatic patients studied. However, higher levels of MUC5AC and lower levels of MUC5B were observed in patients with bronchial hypersecretion in comparison with asthmatics without bronchial hypersecretion but without significant differences (probably, due to the large SD of the values of this mucin). Mucin levels were not correlated with variables such as age, body mass index (BMI), total blood IgE levels, percentage of eosinophils in blood and sputum, and FeNO levels. A positive correlation between MUC 2 levels and $\mathrm{FEV}_{1} \%(r=0.360 ; p=0.003)$ was found in all patients.

\section{Expression of TLRs 2 and 4 in asthmatic patients with and without bronchial hypersecretion}

Expression of TLRs 2 and 4 in macrophages and neutrophils of IS and peripheral blood in asthmatic patients with and without bronchial hypersecretion is detailed in Table 2. No significant differences were observed between groups in the expression of these receptors in inflammatory cells in the 
Table I Demographic, clinical, and functional characteristics of the patients studied

\begin{tabular}{|c|c|c|c|}
\hline & $\begin{array}{l}\text { Asthma with bronchial } \\
\text { hypersecretion } \\
\mathrm{N}=19\end{array}$ & $\begin{array}{l}\text { Asthma without bronchial } \\
\text { hypersecretion } \\
\mathbf{N}=\mathbf{2 4}\end{array}$ & p-value \\
\hline Age (years) mean (SD) & $62.6(10.7)$ & $48.54(16.9)$ & 0.020 \\
\hline Sex (female) \% & $73 \%$ & $66 \%$ & 0.437 \\
\hline BMI $\left(\mathrm{kg} / \mathrm{m}^{2}\right)$ & $28.6(3.4)$ & $26.9(4.7)$ & 0.182 \\
\hline Nasal polyposis (\%) & $36.8 \%$ & $8.3 \%$ & 0.022 \\
\hline Adult asthma diagnosis (\%) & $79 \%$ & $54 \%$ & 0.084 \\
\hline Patients with positive skin prick test (\%) & $31.6 \%$ & $83.4 \%$ & 0.001 \\
\hline Patients with ACT $<20(\%)$ & $73.7 \%$ & $8.3 \%$ & 0.000 \\
\hline Mini-AQLQ, mean (SD) & $4.04(3.1)$ & $1.95(2.1)$ & 0.023 \\
\hline Number of visits to the ER in the past 12 months, mean (SD) & $3.6(3.5)$ & $1.5(2.2)$ & 0.020 \\
\hline Number of courses of $\mathrm{OC}$ received in the last 12 months, mean (SD) & $4.2(3.8)$ & $0.75(1.2)$ & 0.001 \\
\hline Patients with beclomethasone (or equivalent ICS) $\geq 800 \mu \mathrm{g} /$ day (\%) & $78.94 \%$ & $54.16 \%$ & 0.047 \\
\hline Severe asthma (\%) & $94.7 \%$ & $29.2 \%$ & 0.000 \\
\hline $\mathrm{FEV}_{1}(\mathrm{~L})$, mean $(\mathrm{SD})$ & $\mathrm{I} .84(0.7)$ & $2.54(1.0)$ & 0.013 \\
\hline $\mathrm{FEV}_{1} / \mathrm{FVC} \%$, mean (SD) & $60.1(13.4)$ & $69.8(8.1)$ & 0.010 \\
\hline FeNO (ppB), mean (SD) & $32.74(19.8)$ & $37.37(30.4)$ & 0.556 \\
\hline \multirow[t]{4}{*}{ Inflammatory phenotype in induced sputum (\%) } & Paucigranulocytic: $31.5 \%$ & Paucigranulocytic: 29.2 & 0.978 \\
\hline & Neutrophilic: $15.8 \%$ & Neutrophilic: $20.8 \%$ & \\
\hline & Eosinophilic: $47.4 \%$ & Eosinophilic: $45.8 \%$ & \\
\hline & Mixed: $5.3 \%$ & Mixed: $4.2 \%$ & \\
\hline Blood total lgE (Ul/mL), mean (SD) & $113.4(173)$ & $448(536)$ & 0.007 \\
\hline Blood albumin $(g / L)$, mean $(S D)$ & $41(2.7)$ & $44(2.3)$ & 0.000 \\
\hline
\end{tabular}

Notes: Values are mean \pm standard deviation (SD) or percentage, as indicated. Bold figures represent $p<0.05$.

Abbreviations: ACT, Asthma Control Test; BMI, body mass index; ER, emergency room; FeNO, exhaled nitric oxide fraction; FEV second; FVC, forced vital capacity; ICS, inhaled corticosteroids; IgE, total immunoglobulin E; mini-AQLQ, Asthma Quality of Life Questionnaire; OC, oral corticosteroids.

sputum or peripheral blood. A higher intensity of TLR-4 fluorescence was observed in monocytes [2.04 MFI (1.06) vs 1.2 MFI (0.63); $p=0.003$ ] and in peripheral blood neutrophils [2.34 MFI (0.53) vs $1.68 \mathrm{MFI}(0.66) ; p=0.000]$ of hypersecretory patients versus non-hypersecretors.

\section{Discussion}

The main results of the present study were: first, asthmatic patients with bronchial hypersecretion had clinical features different from those without bronchial hypersecretion. Second, mucin expression in IS of these patients did not differ from those who did not present with bronchial hypersecretion. Third, innate immunity, specifically TLRs 2 and 4, did not seem to be involved in the differences between these patients.

Bronchial hypersecretion is a subject that is rarely included as a factor of poor prognosis in patients with asthma and, therefore, few studies consider this variable in patients. ${ }^{1}$ In this study, asthmatic patients with bronchial hypersecretion were characterized by older age, greater severity of asthma, more emergency room visits and use of courses of oral corticosteroids for exacerbations, a greater degree of nasal polyposis, a non-allergic eosinophilic phenotype, poor control of the disease, and a poorer quality of life. This profile with more severe, non-allergic, eosinophilic asthma, with nasal polyposis could overlap with a type of eosinophilic asthma having a pathophysiological mechanism that is yet to be determined, and differs from typical allergic eosinophilic asthma mediated by a Th2 response. Based on these findings, Brusselle et $\mathrm{al}^{27}$ suggest two different roads leading to the eosinophilic inflammation of the airways in asthma. In the allergic type, the protagonist cells would be dendritic cells and CD4+ T cells, with the induction of Th2 cells and production of IL-4, -5, and -13. In non-allergic eosinophilic type, pollution, microbes, and glycolipids could induce the release of cytokines derived from the epithelium, including IL-33, IL-25, and TSLP (epithelial cytokine of the thymic stromal lymphopoietin), which would increase the number of group 2 innate lymphoid cells (ILC2s) in an antigen-dependent manner through their respective receptors (IL-17RB, ST2, and TSLP). Activation of the ILC2s would produce high amounts of IL-5 and IL-13, which in turn would lead to eosinophilia and airway hyperresponsiveness. Along the same lines, Wenzel, ${ }^{28}$ in an article published in Nature Medicine, describes patients who have asthma with persistent eosinophilia despite receiving steroid treatment, and these patients were associated with greater disease severity, beginning in 
Table 2 Mucins levels in induced sputum and expression of TLRs 2 and 4 in asthmatic patients distributed in two groups according to the presence or absence of bronchial hypersecretion

\begin{tabular}{|c|c|c|c|}
\hline & $\begin{array}{l}\text { Asthma with bronchial } \\
\text { hypersecretion } \\
\mathrm{N}=19\end{array}$ & $\begin{array}{l}\text { Asthma without bronchial } \\
\text { hypersecretion } \\
\mathrm{N}=24\end{array}$ & $p$-value \\
\hline \multicolumn{4}{|l|}{ Mucins } \\
\hline MUCI (ng/mL) & II $5.22(45.1)$ & II 4.26 (7I.5) & 0.963 \\
\hline MUC2 (ng/mL) & $21.66(22.8)$ & $27.04(16.6)$ & 0.429 \\
\hline MUC5AC (ng/mL) & $707.42(1515.7)$ & $189.25(306.1)$ & 0.185 \\
\hline MUC5B (ng/mL) & $0.91(2.6)$ & $\mathrm{I} .55(5 . \mathrm{I})$ & 0.647 \\
\hline \multicolumn{4}{|c|}{ Expression of TLRs 2 and 4} \\
\hline \multicolumn{4}{|c|}{ Percentage of cells expressing TLRs2 } \\
\hline \multicolumn{4}{|c|}{ Monocytes (blood) } \\
\hline - Cells (\%) & $77.84(29.6)$ & $72.91(34.6)$ & 0.625 \\
\hline - MFI & $2.4(1.1)$ & $2.3(1.3)$ & 0.869 \\
\hline \multicolumn{4}{|c|}{ Neutrophils (sputum) } \\
\hline - Cells (\%) & $\mathrm{I} .0 \mathrm{I}(\mathrm{I} . \mathrm{I})$ & $1.16(1.5)$ & 0.095 \\
\hline - MFI & $2.19(1.1)$ & $2.63(1.1)$ & 0.642 \\
\hline \multicolumn{4}{|c|}{ Macrophages (sputum) } \\
\hline - Cells (\%) & $27.44(16.6)$ & $17.12(15.1)$ & 0.944 \\
\hline - MFI & $2.55(1.8)$ & $1.99(0.9)$ & 0.682 \\
\hline \multicolumn{4}{|c|}{ Percentage of cells expressing TLRs 4} \\
\hline \multicolumn{4}{|c|}{ Neutrophils (blood) } \\
\hline - Cells (\%) & $2.14(1.3)$ & $1.92(2.5)$ & 0.758 \\
\hline - MFI & $2.34(0.5)$ & $1.68(0.6)$ & 0.002 \\
\hline \multicolumn{4}{|c|}{ Monocytes (blood) } \\
\hline - Cells (\%) & $50.58(23.3)$ & $47.83(25.8)$ & 0.720 \\
\hline - MFI & $2.04(1.1)$ & $1.2(0.6)$ & 0.003 \\
\hline \multicolumn{4}{|c|}{ Neutrophils (sputum) } \\
\hline - Cells (\%) & $3.24(2.7)$ & $7.84(11.7)$ & 0.098 \\
\hline - MFI & $3.27(1.7)$ & $3.16(0.9)$ & 0.096 \\
\hline \multicolumn{4}{|c|}{ Macrophages (sputum) } \\
\hline - Cells (\%) & $41.73(20.8)$ & $46.74(19.6)$ & 0.804 \\
\hline - MFI & $5.54(2.3)$ & $8.14(3.8)$ & 0.072 \\
\hline
\end{tabular}

Notes: Values are mean percentage and standard deviation (SD) for mucins and cell counts, and mean (SD) for MFI. Bold figures represent $p<0.05$. Abbreviations: MUC, mucins; MFI, mean of fluorescence intensity; TLRs, toll-like receptors.

late age, little family history of asthma, intolerance at times to nonsteroidal anti-inflammatory drugs (NSAIDs), sinusitis, polyps, and a less allergic component (bronchial hypersecretion was not assessed in this study). Lack of allergy in these patients suggests a different mechanism from Th2, and is probably more complex than the typical early-onset allergic phenotype. ${ }^{28}$ The results of our study were related to this profile of patients with characteristics presented in our work by asthmatic patients with bronchial hypersecretion - that is, patients with greater severity, older, more polyposis, and with an inflammatory phenotype predominantly eosinophilic, not allergic. These results, despite describing an asthma phenotype comparable to those mentioned previously in the literature, require further studies to confirm this relationship. In these studies, we propose the inclusion of the variable of bronchial hypersecretion of mucus in asthmatic patients in their analysis as a factor of worse prognosis.
Several studies have shown that the mucus of asthmatic patients shows greater viscosity than that of patients with chronic obstructive pulmonary disease (COPD) and cystic fibrosis $(\mathrm{CF}) ; ;^{3,29}$ in addition, they reported that this difference lies in the fact that they have a different mucin profile. MUCs are glycoproteins that are part of the airway mucus; they are responsible for its viscoelastic properties and play an important antimicrobial and anti-inflammatory role in acting as a first immunological line of defence. ${ }^{5}$ MUCs are composed of alpha and beta subunits that undertake cell adhesion and cellular signaling. In patients with COPD, MUCs present with elevated levels, specifically MUC5AC, MUC5B, and MUC 2, unlike in asthmatic patients where MUC5AC and MUC5B show values tending to normal and only a small amount of MUC2 is present. ${ }^{3,29}$ In our study, no significant differences were observed in the expression of mucin among asthmatic patients who did or did not present 
with bronchial hypersecretion, and taking both groups of asthmatic patients as a whole, the results agree with the literature, with the MUC1 being very high, followed by the MUC2 being slightly elevated, and MUC5AC and MUC5B being inside the reference values. On the other hand, it should be noted that we observed higher levels of MUC5AC and lower levels of MUC5B in patients with bronchial hypersecretion in comparison with asthmatics without bronchial hypersecretion (non-significant changes). Some reports have described technical difficulties in quantifying sputum mucins by direct-binding ELISAs due to high levels of nonmucin proteins that may interfere with the immobilization of the mucins on the plate ${ }^{30}$; this point may be a limitation for consideration in our study.

Recently, it has been shown that MUC1 plays an antiinflammatory role in bacterial infection of the airways, and this effect involves the participation of TLRs. ${ }^{17}$ It is suspected that the MUC1, in addition to suppressing the activation of NF-KB (at the TRAF6-TAK1 complex level), can stimulate these TLR signals. Thus, it is known that other signals (TLRs 2, 3, 4, 7, and 9) are also suppressed by MUC1. TLRs are responsible for the recognition of pathogen-associated molecular patterns; therefore, both proteins (MUC and TLRs) are involved primarily in local defense against respiratory infections. TLRs have been related to both neutrophilic and allergic asthma, specifically TLRs 2 and 4, and activation of TLRs. ${ }^{6}$ In our study, we found high levels of MUC1 expression in both groups of asthmatic patients, but no significant differences between the groups with and without bronchial hypersecretion were observed.

Our study has some limitations: 1) we did not purify mucins from nonmucin proteins, nor use cocktails of antibodies to maximize epitope regnition; ${ }^{30}$ 2) the effect of inhaled corticosteroids on expression of TLRs is unknown; 3) we only analyzed receptors that are expressed on the cell surface (TLRs 4 and 2); 4) this was a descriptive study with a small sample of patients, but has several strengths as compared to previous reports. This was a comprehensive study in a large sample using multiple techniques to assess various aspects of the disease, whereas other studies to date have focused on specific or partial aspects. Furthermore, it is the first study measuring mucins and TLRs in the sputum of asthmatic patients and all the measurements were made on the same day in all patients (clinical, functional, and inflammatory variables).

\section{Conclusion}

In summary, we can conclude that asthmatic patients with bronchial hypersecretion have a more severe and uncontrolled disease, an inflammatory phenotype that is predominantly non-allergic, and present with a poor quality of life. Regardless of whether innate immunity plays a role in the pathogenesis of this type of asthma with bronchial hypersecretion, the present study failed to demonstrate an abnormality in the mucin profile and in TLRs 2 and 4 in sputum and blood. Because this study shows that asthmatic patients with bronchial hypersecretion present with a poor prognosis, from a clinical standpoint, this variable should be elicited in the patient's history.

\section{Highlights}

- Despite the clinical repercussions of bronchial mucus hypersecretion in the asthmatic patient, this variable has been poorly studied.

- To date, the relationship of innate immunity with the presence of bronchial mucus hypersecretion in asthmatic patients is unknown.

- TLRs and mucins play an important role in defense against pathogens. Several alterations of these proteins have been described as part of the pathophysiological mechanism of some asthma phenotypes.

- The possible recognition of a new endotype of asthma, mediated by the alteration of TLRs and mucins, especially in asthma with bronchial mucus hypersecretion, could open new therapeutic expectations, especially in a type of asthma with a traditional low response to conventional treatment of the illness.

\section{Acknowledgments}

This research was supported by grants from the Spanish Respiratory Society (SEPAR) and Catalan Pulmonology Foundation (FUCAP), Barcelona, Spain. The authors are grateful to Lorena Soto for editing the manuscript. Data sharing not applicable to this article as no datasets were generated or analyzed during the current study.

\section{Author contributions}

ACL generated the hypothesis, designed the study, enrolled patients, and wrote the first draft of the manuscript; VP and ACL collected patient clinical data, built the database, and conducted statistical analysis; JG assessed spirometry data and FeNO determinations; MT and AB induced sputum and undertook extraction of peripheral blood and skin prick test; EM processed sputum and performed the inflammatory cell counts and quantification and determination the TLRs and mucins; SV participated in data interpretation of cytometry and ELISA; and OS participated in data interpretation and 
manuscript writing. All authors contributed toward data analysis, drafting and revising the paper and agree to be accountable for all aspects of the work.

\section{Disclosure}

The authors report no conflicts of interest in this work.

\section{References}

1. Weatherall M, Travers J, Shirtcliffe PM, et al. Distinct clinical phenotypes of airways disease defined by cluster analysis. Eur Respir J. 2009;34(4):812-818.

2. Rogers DF. Physiology of airway mucus secretion and pathophysiology of hypersecretion. Respir Care. 2007;52(9):1134-1149.

3. Rogers DF. Airway mucus hypersecretion in asthma: an undervalued pathology? Curr Opin Pharmacol. 2004;4(3):241-250.

4. Evans CM, Kim K, Tuvim MJ, Dickey BF. Mucus hypersecretion in asthma: causes and effects. Curr Opin Pulm Med. 2009;15(1):4-11.

5. Voynow JA, Rubin BK. Mucins, mucus and sputum. Chest. 2009; 135(2):505-512.

6. Crespo-Lessmann A, Juárez-Rubio C, Plaza-Moral V. Role of toll-like receptors in respiratory diseases. Arch Bronconeumol. 2010;46(3):135142. [Article in Spanish]

7. Wang K, Wen FQ, Xu D. Mucus hypersecretion in the airway. Chin Med J (Engl). 2008;121(7):649-652.

8. Morinaga Y, Yanagihara K, Miyashita N, et al. Azithromycin, clarithromycin and telithromycin inhibit MUC5AC induction by Chlamydophila pneumoniae in airway epithelial cells. Pulm Pharmacol Ther. 2009;22(6):580-586.

9. Kraft M, Adler KB, Ingram JL, et al. Mycoplasma pneumoniae induces airway epithelial cell expression of MUC5AC in asthma. Eur Respir J. 2008;31(1):43-46.

10. Simpson JL, Grissell TV, Douwes J, Scott RJ, Boyle MJ, Gibson PG. Innate immune activation in neutrophilic asthma and bronchiectasis. Thorax. 2007;62(3):211-218.

11. Beckett EL, Phipps S, Starkey MR, et al. TLR2, but not TLR4, is required for effective host defence against Chlamydia respiratory tract infection in early life. PLoS One. 2012;7(6):e39460.

12. Kato K, Lu W, Kai H, Kim KC. Phosphoinositide 3-kinase is activated by MUC1 but not responsible for MUC1-induced suppression of toll-like receptor 5 signalling. Am J Physiol Lung Cell Mol Physiol. 2007;293(3):L686-L692.

13. Ueno K, Koga T, Kato K, et al. MUC1 mucin is a negative regulator of tolllike receptor signalling. Am J Respir Cell Mol Biol. 2008;38(3):263-268.

14. GINA. Global strategy for asthma management and prevention. Available from: http://www.ginasthma.org/. 2014. Accessed November 3, 2015.
15. Vega JM, Badia X, Badiola C, et al; Covalair Investigator Group. Validation of the Spanish version of the Asthma Control Test (ACT). J Asthma. 2007;44(10):867-872.

16. Sanjuàs C, Alonso J, Sanchís J, et al. The quality-of-life questionnaire with asthma patients: the Spanish version of the Asthma Quality of Life Questionnaire. Arch Bronconeumol. 1995;31(5):219-226. [Article in Spanish]

17. American Thoracic Society; European Respiratory Society. ATS/ERS Recommendations for standardized procedures for the online and offline measurement of exhaled lower respiratory nitric oxide and nasal nitric oxide. Am J Respir Crit Care Med. 2005;171(8):912-930.

18. Guía Española para el Manejo del Asma (GEMA 4.0). Available from: http://www.gemasma.com. Accessed November 3, 2015.

19. García-Río F, Calle M, Burgos F, Casan P, del Campo F, Gáldiz JB, et al. Spirometry. Spanish Society of Pulmonology and Thoracic Surgery (SEPAR). Arch Bronconeumol. 2013;49(9):388-401.

20. Roca J, Sanchis J, Agusti-Vidal A, et al. Spirometric reference values from a Mediterranean population. Bull Eur Physiopathol Respir. 1986;22(3):217-224.

21. Paggiaro PL, Chanez P, Holz O, et al. Sputum induction. Eur Respir $J$ Suppl. 2002;37:3s-8s.

22. Vidal S, Bellido-Casado J, Granel C, Crespo A, Plaza V, Juárez C. Flow cytometry analysis of leukocytes in induced sputum from asthmatic patients. Inmunobiology. 2012;217(7):692-697.

23. Pizzichini E, Pizzichini MM, Efthimiadis A, et al. Indices of airway inflammation in induced sputum: reproducibility and validity of cell and fluid-phase measurements. Am J Respir Crit Care Med. 1996;154(2 Pt 1):308-317.

24. Magnussen H, Holz O, Sterk PJ, Hargreave FE. Noninvasive methods to measure airway inflammation: future considerations. Eur Respir J. 2000;16(6):1175-1179.

25. Pepys J. Skin testing. Br J Hosp Med. 1975;14:412-414.

26. BTS. British Thoracic Society, Scottish Intercollegiate Guidelines Network. British guideline on the management of asthma. Available from: http://www.sign.ac.uk/pdf/sign101.pdf. 2014. Accessed November 3, 2015.

27. Brusselle GG, Maes T, Bracke KR. Eosinophils in the spotlight: eosinophilic airway inflammation in nonallergic asthma. Nat Med. 2013;19(8):977-979.

28. Wenzel SE. Asthma phenotypes: the evolution from clinical to molecular approaches. Nat Med. 2012;18(5):716-725.

29. Jinnai M, Niimi A, Ueda T, et al. Induced sputum concentrations of mucin in patients with asthma and chronic cough. Chest. 2010;137(5): $1122-1129$.

30. Lachowicz-Scroggins ME, Yuan S, Kerr SC, et al. Abnormalities in MUC5AC and MUC5B protein in airway mucus in asthma. Am J Respir Crit Care Med. 2016;194(10):1296-1299.
Journal of Asthma and Allergy

\section{Publish your work in this journal}

The Journal of Asthma and Allergy is an international, peer-reviewed open access journal publishing original research, reports, editorials and commentaries on the following topics: Asthma; Pulmonary physiology; Asthma related clinical health; Clinical immunology and the immunological basis of disease; Pharmacological interventions and

\section{Dovepress}

new therapies. This journal is included in PubMed. The manuscript management system is completely online and includes a very quick and fair peer-review system, which is all easy to use. Visit http://www. dovepress.com/testimonials.php to read real quotes from published authors. 